A. B. Kharazishvili, Institute of Applied Mathematics, University of Tbilisi, University str. 2, 380043 Tbilisi 43, Georgia.

\title{
SOME REMARKS ON ADDITIVE PROPERTIES OF INVARIANT $\sigma$-IDEALS ON THE REAL LINE
}

\begin{abstract}
We consider some additive properties of invariant $\sigma$-ideals and $\sigma-$ algebras of subsets of the real line $\mathbb{R}$. In particular, we generalize two classical Sierpinski results, concerning additive properties of measure and category on $\mathbb{R}$, to a large class of invariant $\sigma$-ideals and $\sigma$-algebras.
\end{abstract}

Let $M$ be a class of subsets of the real line $\mathbb{R}$. We shall say that this class is invariant if the following two conditions hold.

1) For each set $X \in M$ and for each element $r \in \mathbb{R}$ we have $r+X \in M$.

2) For any set $X \in M$ and for any non-zero rational number $q$ we have $q X \in M$.

In other words, our class $M$ is invariant if and only if it is invariant under the group $G$ of all affine transformations $g$ of $\mathbb{R}$ which can be represented in the form

$$
g(z)=q z+r \quad(z \in \mathbb{R}),
$$

where $q$ is a non-zero rational number and $r$ is a real number.

In our further considerations we shall often use the well known fact that all vector spaces over the rationals of the cardinality of the continuum are isomorphic. Hence, all of them are isomorphic to $\mathbb{R}$ considered as a vector space over rationals. Note that the properties we are interested in are preserved under isomorphisms of such vector spaces.

Key Words: real line, invariant $\sigma$-ideal, invariant $\sigma$-algebra, countable chain condition, Hamel base

Mathematical Reviews subject classification: Primary: 28A05, 28D05

Received by the editors February 16, 1995 
Let $I$ be an invariant $\sigma$-ideal of subsets of the real line $\mathbb{R}$ containing all oneelement subsets of $\mathbb{R}$. For example, $I$ may be the $\sigma$-ideal $\mathbb{L}$ of all Lebesgue measure zero subsets of $\mathbb{R}$ or $I$ may be the $\sigma$-ideal $\mathbb{K}$ of all first category subsets of $\mathbb{R}$. In this paper we shall consider some questions connected with the existence of two sets $X$ and $Y$ belonging to $I$ such that their vector sum

$$
X+Y=\{x+y: x \in X, y \in Y\}
$$

does not belong to $I$. We begin with the following simple proposition.

Proposition 1 Let I be an arbitrary invariant $\sigma$-ideal of subsets of $\mathbb{R}$, then the following are equivalent.

1) There exist two sets $X \in I$ and $Y \in I$ such that $X+Y \notin I$.

2) There exists a set $X \in I$ such that $X+X \notin I$.

3) There exists a linearly independent (over the field $\mathbb{Q}$ of all rational numbers) set $X \in I$ such that lin $(X) \notin I$, where lin $(X)$ denotes the vector space over $\mathbb{Q}$ generated by the set $X$.

4) There exists a set $X \in I$ such that $\operatorname{lin}(X) \notin I$.

Proof. Obviously, it is sufficient to prove the following implications

$$
\text { 1) } \Rightarrow 2) \Rightarrow 3) \Rightarrow 4) \Rightarrow 1) \text {. }
$$

Suppose that relation 1) holds. Then there are sets $A \in I$ and $B \in I$ such that $A+B \notin I$. Put $X=A \cup B$. It is clear that the set $X$ satisfies relation $2)$. Thus, implication 1) $\Rightarrow 2$ ) is established.

Now suppose that relation 2) holds. Then there exists a set $Y \in I$ such that $Y+Y \notin I$. Let $X$ be a maximal (with respect to inclusion) linearly independent (over the field $\mathbb{Q}$ ) subset of $Y$. Then it is obvious that $\operatorname{lin}(X)=$ $\operatorname{lin}(Y)$ and $X \in I$. Furthermore, we have

$$
Y+Y \subseteq \operatorname{lin}(Y)=\operatorname{lin}(X), \quad \operatorname{lin}(X) \notin I .
$$

Consequently, the set $X$ satisfies relation 3 ). Thus, implication 2) $\Rightarrow 3$ ) is established.

Implication 3$) \Rightarrow 4$ ) is trivial.

Now suppose that relation 4) holds. Let $D \in I$ be a subset of the real line such that $\operatorname{lin}(D) \notin I$. Evidently, we can write

$$
\operatorname{lin}(D)=\cup\left\{D_{n}: 0<n<\omega\right\},
$$


where $D_{n}$ denotes the vector sum

$$
\mathbb{Q} D+\mathbb{Q} D+\ldots+\mathbb{Q} D
$$

in which the number of summands is equal to $n$. Since $\operatorname{lin}(D)$ does not belong to $I$ there exists the least natural number $m$ such that the set $D_{m}$ also does not belong to $I$. It is obvious that $m>1$. If we put $X=\mathbb{Q} D, Y=D_{m-1}$, then it is easy to check that $X \in I, \quad Y \in I, \quad X+Y \notin I$. Therefore, the sets $X$ and $Y$ satisfy relation 1 ). Thus, implication 4$) \Rightarrow 1$ ) is established, and the proof of Proposition 1 is complete.

In connection with Proposition 1 let us give an example of a non-trivial invariant $\sigma$-ideal on $\mathbb{R}$ for which all relations 1) - 4) are false.

Example 1. Let $E$ be an arbitrary infinite-dimensional separable Hilbert space over the field $\mathbb{R}$. We define a class $J$ of subsets of $E$ by the equality

$$
J=\{X \text { : there exists a countable covering of } X \text { by compact sets in } E\} .
$$

Obviously, $J$ is an invariant $\sigma$-ideal of subsets of $E$ for which we have

$$
J+J=\{X+Y: X \in J, Y \in J\}=J .
$$

If we consider the space $E$ and the real line $\mathbb{R}$ as vector spaces over the field $\mathbb{Q}$, then it is not difficult to show, using Hamel bases in these spaces, that $E$ and $\mathbb{R}$ are isomorphic. Take any isomorphism $f: E \rightarrow \mathbb{R}$ and put $I=f(J)$. Then it is clear that the class $I$ of subsets of $\mathbb{R}$ satisfies the following relations.

(1) $I$ is an invariant $\sigma$-ideal on $\mathbb{R}$.

(2) $I+I=I$.

(3) There exists a base $\Phi$ of the $\sigma$-ideal $I$ such that $\operatorname{card}(\Phi)=\mathfrak{c},(\forall X \in$ $I)(\exists Y \in \Phi)(X \subseteq Y \& \operatorname{card}(Y \backslash X)=\mathfrak{c})$, where $\mathfrak{c}$ denotes the cardinality of the continuum.

Applying the Sierpinski-Erdos Duality Principle (See, for instance, [1, Chapter 19], or [2, pp. 98-101].) and taking into account relation (3), we conclude that if the Continuum Hypothesis holds, then the $\sigma$-ideal $I$ is isomorphic in the set-theoretical sense to the $\sigma$-ideal $\mathbb{L}$ of all Lebesgue measure zero subsets of $\mathbb{R}$ (or to the $\sigma$-ideal $\mathbb{K}$ of all first category subsets of $\mathbb{R}$ ). On the other hand, it is well-known that $\mathbb{L}+\mathbb{L} \neq \mathbb{L}$ and $\mathbb{K}+\mathbb{K} \neq \mathbb{K}$. It immediately follows from this fact that there does not exist a linear isomorphism (over $\mathbb{Q}$ ) between $\sigma$-ideals $I$ and $\mathbb{L}$ or between $\sigma$-ideals $I$ and $\mathbb{K}$. In connection with this observation let us recall the following old, still unsolved problem concerning the Sierpinski-Erdos 
Duality Principle. Assuming the Continuum Hypothesis, does there exist a linear (over $\mathbb{Q}$ ) Sierpinski-Erdos Duality between two classical $\sigma$-ideals $\mathbb{L}$ and $\mathbb{K}$ ?

Finally, let $S$ be the $\sigma$-algebra of subsets of $\mathbb{R}$ generated by the $\sigma$-ideal $I$ constructed above. Evidently, $S$ is an invariant $\sigma$-algebra containing $I$ such that $S+S=S$. It is worth remarking here that for the pair $(I, S)$ the countable chain condition holds. Recall that $(I, S)$ satisfies the countable chain condition if for every uncountable family $\left\{X_{\xi}: \xi<\omega_{1}\right\}$ of pairwise disjoint elements of $S$ there always exists an element $X_{\xi}$ belonging to $I$. (From this definition it follows also that all sets $X_{\xi}$, except a countable number of them, belong to I.) In our case a much stronger condition holds. Namely, for any two sets $X \in S \backslash I$ and $Y \in S \backslash I$ the intersection $X \cap Y$ also belongs to $S \backslash I$ and hence is a non-empty set.

Remark 1. Let $\mathbb{R}^{2}$ be the Euclidean plane. Denote by $J$ the class of all sets $X$ such that there exists a countable covering of $X$ by straight lines lying in $\mathbb{R}^{2}$ and parallel to the line $\{0\} \times \mathbb{R}$. Obviously, $J$ is an invariant $\sigma$-ideal of subsets of $\mathbb{R}^{2}$ and $J+J=J$. Consider $\mathbb{R}^{2}$ and $\mathbb{R}$ as vector spaces over the field $\mathbb{Q}$. Applying Hamel bases again it is easy to see that these vector spaces are isomorphic. Take any isomorphism $f: \mathbb{R}^{2} \rightarrow \mathbb{R}$ and put $I=f(J)$. Then $I$ also satisfies relations (1) - (3) of Example 1.

Now let $S$ be an arbitrary invariant $\sigma$-algebra of subsets of the real line $\mathbb{R}$ and let $I$ be an arbitrary invariant $\sigma$-ideal of subsets of $\mathbb{R}$ such that $I \subseteq S$. Suppose that there exist sets $A \in I$ and $B \in I$ satisfying the relation $A+B \notin I$. Then the following question arises in a natural way. Do there exist sets $X \in I$ and $Y \in I$ such that $X+Y \notin S$ ?

In the general case the answer to the posed question is negative. This can be shown by the following "geometric" example.

Example 2. Let us consider the three-dimensional Euclidean space $\mathbb{R}^{3}$ and let us define two classes of sets

$$
\begin{aligned}
& J_{1}=\left\{X \text { : there exists a countable covering of } X \text { by straight lines in } \mathbb{R}^{3}\right\}, \\
& J_{2}=\left\{X \text { : there exists a countable covering of } X \text { by planes in } \mathbb{R}^{3}\right\} .
\end{aligned}
$$

Obviously, $J_{1}$ and $J_{2}$ are invariant $\sigma$-ideals of subsets of $\mathbb{R}^{3}$ and $J_{1} \subseteq J_{2}$. Let $T$ be the $\sigma$-algebra of subsets of $\mathbb{R}^{3}$ generated by the $\sigma$-ideal $J_{2}$. Then $T$ is an invariant class of sets as well. It is easy to check that for the pair $\left(J_{1}, T\right)$ the following relations hold.

1) $\left(J_{1}, T\right)$ does not satisfy the countable chain condition.

2) $J_{1}+J_{1} \subseteq J_{2} \subseteq T$. 
3) There exist two sets $X \in J_{1}$ and $Y \in J_{1}$ such that $X+Y \notin J_{1}$.

Now let us consider the space $\mathbb{R}^{3}$ and the real line $\mathbb{R}$ as vector spaces over the field $\mathbb{Q}$. Since these vector spaces are isomorphic, we can take an isomorphism $f: \mathbb{R}^{3} \rightarrow \mathbb{R}$ and put $I=f\left(J_{1}\right)$ and $S=f(T)$. Then it is clear that for the pair $(I, S)$ the relations analogous to relations 1$)-3)$ hold. In particular, we have $I+I \subseteq S$ and there exist two sets $X \in I$ and $Y \in I$ such that $X+Y \notin I$.

Of course, the pair $(I, S)$ of Example 2 does not satisfy the countable chain condition. Let us note that if the countable chain condition holds, then we have an essentially different situation. In order to establish this fact we need two auxiliary propositions.

Lemma 1 Let $E$ be a basic set, let $I$ be a $\sigma$-ideal of subsets of $E$ and let $S$ be a $\sigma$-algebra of subsets of $E$ containing the ideal $I$. Suppose also that the countable chain condition holds for the pair $(I, S)$. Let $m>0$ be a fixed natural number and let $\left\{X_{\alpha}: \alpha<\omega_{1}\right\}$ be an uncountable family of elements of $S$ such that for every $m$-element subset $A$ of $\omega_{1}$ we have

$$
\cap\left\{X_{\alpha}: \alpha \in A\right\} \in I .
$$

Then there exists an uncountable subset $B$ of $\omega_{1}$ such that

$$
\left\{X_{\beta}: \beta \in B\right\} \subseteq I .
$$

The proof of Lemma 1 is not difficult. We can use here induction on $m$. The cases $m=1$ and $m=2$ are trivial. Assume that the assertion of Lemma 1 is true for $m-1>1$. Let $Y_{A}=\cap\left\{X_{\alpha}: \alpha \in A\right\}$. Fix a subset $A$ of $\omega_{1}$ with $\operatorname{card}(A)=m-2$ and consider the family of sets $\left\{X_{\xi} \cap Y_{A}: \xi \in \omega_{1} \backslash A\right\}$. It is clear that the intersection of any two sets from this family belongs to the ideal I. Consequently, there exists an ordinal $\xi(A)<\omega_{1}$ such that $X_{\xi} \cap Y_{A} \in I$ for all ordinals $\xi$ satisfying the inequalities $\xi(A)<\xi<\omega_{1}$. Using this fact and applying the regularity of $\omega_{1}$ we can recursively define an injective subfamily

$$
X_{\xi(0)}, X_{\xi(1)}, \ldots, X_{\xi(\alpha)}, \ldots\left(\alpha<\omega_{1}\right)
$$

such that, for every $(m-1)$-element subset $A$ of $\omega_{1}$, the relation

$$
\cap\left\{X_{\xi(\alpha)}: \alpha \in A\right\} \in I
$$

holds. Thus, by induction, Lemma 1 is proved.

We can also immediately deduce from this lemma that, actually, we have

$$
\operatorname{card}\left(\left\{\alpha<\omega_{1}: X_{\alpha} \notin I\right\}\right) \leq \omega .
$$


Lemma 2 Let $D$ be an arbitrary linearly independent (over the field $\mathbb{Q}$ ) subset of the real line $\mathbb{R}$ and let $n>0$ be a fixed natural number. Consider the set $D_{n}$ defined in the proof of Proposition 1. Then there exists an uncountable family $\left\{z_{\xi}: \xi<\omega_{1}\right\}$ of elements of $\mathbb{R}$ such that the family

$$
\left\{D_{n}+z_{\xi}: \xi<\omega_{1}\right\}
$$

consists of pairwise disjoint sets. Moreover, if $D$ is an uncountable set, then we may assume that all $z_{\xi}\left(\xi<\omega_{1}\right)$ belong to $\operatorname{lin}(D)$.

The proof of Lemma 2 also is not difficult. Indeed, without loss of generality we can suppose that $D$ is an uncountable set. For each element $z \in \operatorname{lin}(D)$ we have the unique representation

$$
z=q_{1} d_{1}+q_{2} d_{2}+\ldots+q_{m} d_{m}
$$

where $q_{1}, q_{2}, \ldots, q_{m}$ are some non-zero rational numbers and $d_{1}, d_{2}, \ldots, d_{m}$ are some pairwise distinct elements of $D$. Let us put $m(z)=m$. By transfinite recursion, it is not difficult to define a family $\left\{z_{\xi}: \xi<\omega_{1}\right\}$ of elements of $\operatorname{lin}(D)$ such that $m\left(z_{\xi}-z_{\zeta}\right)>2 n$ for any two ordinals $\xi<\omega_{1}, \zeta<\omega_{1}, \xi \neq \zeta$. Now it is easy to check that this family is a required one.

In connection with Lemma 2 see also two classical papers [5] and [6].

Proposition 2 Let $I$ be an invariant $\sigma$-ideal of subsets of $\mathbb{R}$, let $S$ be an invariant $\sigma$-algebra of subsets of $\mathbb{R}$ containing $I$ and let the pair $(I, S)$ satisfy the countable chain condition. Then the following are equivalent.

1) There exist sets $X \in I$ and $Y \in I$ such that $X+Y \notin I$.

2) There exists a set $X \in I$ such that $X+X \notin S$.

3) There exists a linearly independent (over the field $\mathbb{Q}$ ) set $X \in I$ such that $\operatorname{lin}(X) \notin S$.

4) There exists a set $X \in I$ such that $\operatorname{lin}(X) \notin S$.

Proof. Taking into account Proposition 1 we see that it is sufficient to prove the following implications 1) $\Rightarrow 3$ ) $\Rightarrow 2$ ). Suppose that relation 1) holds, i.e. the ideal $I$ is not closed under the operation of vector sum of elements of $I$. Then, by Proposition 1, there exists a linearly independent (over $\mathbb{Q}$ ) set $Z \in I$ such that $\operatorname{lin}(Z) \notin I$. Since the set $Z$ is uncountable we can represent it as the union of two disjoint sets $V$ and $U$ in such a way that $\operatorname{card}(V)=\omega_{1}$. Hence, $\operatorname{lin}(Z)$ can be represented as the direct sum of vector spaces $\operatorname{lin}(V)$ and 
$\operatorname{lin}(U)$. If we have $\operatorname{lin}(U) \notin S$, then it is nothing to prove. So, suppose that $\operatorname{lin}(U) \in S$. Taking into account the fact that there exists an uncountable family of pairwise disjoint translates of $\operatorname{lin}(U)$, we conclude that $\operatorname{lin}(U) \in I$.

Now let us consider the classical transfinite matrix of Ulam over the basic set $V$, i.e. consider a family

$$
\left\{V_{n, \xi}: n<\omega, \xi<\omega_{1}\right\}
$$

of subsets of $V$ satisfying the following conditions.

(a) For each ordinal $\xi<\omega_{1}$ we have $\operatorname{card}\left(V \backslash \cup\left\{V_{n, \xi}: n<\omega\right\}\right) \leq \omega$;

(b) For every natural number $n$ the family $\left\{V_{n, \xi}: \xi<\omega_{1}\right\}$ consists of pairwise disjoint sets.

The construction of the Ulam matrix mentioned above is presented in many papers and books. (See, e.g., [2, p. 74].) Some non-trivial applications of the Ulam matrix are discussed, for example, in works [1] - [4]. In particular, some applications of this matrix to the theory of invariant (quasiinvariant) measures are discussed in monograph [3, p. 65-68].

Actually, we deal here with another transfinite matrix that is generated by the Ulam matrix. Namely, let us put

$$
W_{n, \xi}=\cup\left\{V_{k, \xi}: k \leq n\right\} \quad\left(n<\omega, \xi<\omega_{1}\right)
$$

and let us consider the family of vector spaces

$$
\operatorname{lin}\left(W_{n, \xi}\right)+\operatorname{lin}(U) \quad\left(n<\omega, \xi<\omega_{1}\right) .
$$

Applying property $(a)$ of the Ulam matrix it is easy to see that for each ordinal $\xi<\omega_{1}$ there exists a natural number $n=n(\xi)$ such that the vector space $\operatorname{lin}\left(W_{n, \xi}\right)+\operatorname{lin}(U)$ does not belong to the ideal $I$. Consequently, there exist a natural number $m$ and an uncountable subset $\Xi$ of $\omega_{1}$ such that all vector spaces

$$
\operatorname{lin}\left(W_{m, \xi}\right)+\operatorname{lin}(U) \quad(\xi \in \Xi)
$$

also do not belong to $I$. Now, taking into account the fact that, the set $V$ is linearly independent over $\mathbb{Q}$ and using property (b) of the Ulam matrix, we see that for any $(m+2)$-element subset $\Theta$ of $\Xi$, the equality

$$
\cap\left\{\operatorname{lin}\left(W_{m, \xi}\right)+\operatorname{lin}(U): \xi \in \Theta\right\}=\operatorname{lin}(U)
$$

holds. Since we have $\operatorname{lin}(U) \in I$, we can apply Lemma 1 here. According to this lemma there exists at least one ordinal $\zeta \in \Xi$ such that the corresponding vector space $\operatorname{lin}\left(W_{m, \zeta}\right)+\operatorname{lin}(U)$ does not belong to the $\sigma$-algebra $S$. (Moreover, we see that there is an uncountable family of ordinals $\zeta \in \Xi$ such that all vector 
spaces $\operatorname{lin}\left(W_{m, \zeta}\right)+\operatorname{lin}(U)$ do not belong to $S$.) Now, if we put $X=W_{m, \zeta} \cup U$, then it is easy to check that the set $X$ satisfies relation 3 ).

It remains to prove implication 3$) \Rightarrow 2$ ). Suppose that relation 3) holds and take any linearly independent (over $\mathbb{Q}$ ) set $D \in I$ such that $\operatorname{lin}(D) \notin S$. Of course, we can write $\operatorname{lin}(D)=\cup\left\{D_{n}: 0<n<\omega\right\}$, where $D_{n}(0<n<\omega)$ are the sets defined in the proof of Proposition 1. Obviously, there exists the least natural number $m$ such that $D_{m} \notin S$, and it is clear that $m>1$. Only two cases are possible.

1. $m$ is an even number, i.e. $m=2 k$. In this case we have $D_{k} \in S$. Taking into account Lemma 2 , we also have $D_{k} \in I$. Let us put $X=D_{k}$. Then

$$
X+X=D_{k}+D_{k}=D_{2 k}=D_{m} \notin S .
$$

Hence, the set $X$ satisfies relation 2).

2. $m$ is an odd number, i.e. $m=2 k+1$. In this case we have $k+1<2 k+1$ and $D_{k+1} \in S$. Applying Lemma 2 again, we get $D_{k+1} \in I$. Now consider the set $D_{2 k+2}$. It is easy to see that this set does not belong to $S$. Indeed, if $D_{2 k+2} \in S$, then by Lemma 2 we obtain $D_{2 k+2} \in I$ and therefore,

$$
D_{2 k+1} \subseteq D_{2 k+2} \in I, \quad D_{2 k+1} \in I \subseteq S
$$

which contradicts the definition of the number $m$. Consequently, $D_{2 k+2} \notin S$. Let us put $X=D_{k+1}$. Then it is clear that $X$ satisfies relation 2).

Example 3. Let $I$ be an invariant $\sigma$-ideal of subsets of the real line $\mathbb{R}$, let $S$ be an invariant $\sigma$-algebra of subsets of $\mathbb{R}$ such that $I \subseteq S$ and let the pair $(I, S)$ satisfy the countable chain condition. Suppose also that the classical Cantor subset $C$ of the real line belongs to the ideal $I$. It is well-known that $C+C=[0,2]$ (see, e.g., [2], p. 202, where a geometric interpretation of this equality is also given). Hence, we have $C+C \notin I$. Applying Proposition 2, we conclude that there exists a set $X \in I$ such that $X+X \notin S$. In particular, we obtain the following two classical results of Sierpinski.

1) $\mathbb{L}+\mathbb{L}$ contains a Lebesgue non-measurable set.

2) $\mathbb{K}+\mathbb{K}$ contains a set without the Baire property.

In connection with Example 3 see also [2, p. 226].

Let $I$ be again an invariant $\sigma$-ideal of subsets of $\mathbb{R}$, let $S$ be an invariant $\sigma$-algebra of subsets of $\mathbb{R}$ containing $I$ and let the pair $(I, S)$ satisfy the countable chain condition. From Proposition 2 it follows, in particular, that the implication

$$
I+I \neq I \Rightarrow S+S \neq S
$$


is true. The next example shows us that the converse implication is not true in general.

Example 4. Recall that Sierpinski constructed, by the method of transfinite recursion, a subset $A$ of $\mathbb{R}$ for which the following conditions hold.

$(*) \operatorname{card}(A)=\operatorname{card}(\mathbb{R} \backslash A)=\mathfrak{c}$.

$(* *)$ For each $r \in \mathbb{R}$ we have $\operatorname{card}((r+A) \triangle A)<\mathfrak{c}$, where the symbol $\triangle$ denotes the operation of symmetric difference of two sets.

The construction of the set $A$ is considered in detail in several books, e.g., [1], [2], [3], and [7]. See, in particular, [2, p. 213]. Moreover, one can find in [2] and [3] various constructions of subsets of $\mathbb{R}$ similar to $A$. Some applications of such subsets to the theory of invariant extensions of the Lebesgue measure are discussed in monograph [3, p. 117].

Notice also that $A$ cannot be a Borel subset of $\mathbb{R}$.

We need here a two-dimensional analog of $A$ with some additional properties. Let $H$ be the group of affine transformations of the Euclidean plane $\mathbb{R}^{2}$ generated by all translations of $\mathbb{R}^{2}$ and all homotheties of $\mathbb{R}^{2}$ with the center $(0,0)$ and with non-zero coefficients. We can define by the method of transfinite recursion a subset $B$ of the Euclidean plane $\mathbb{R}^{2}$ satisfying the following conditions.

1) For every straight line $P$ lying in $\mathbb{R}^{2}$ and parallel to the line $\{0\} \times \mathbb{R}$ we have $\operatorname{card}(P \cap B)<\mathfrak{c}$.

2) For each transformation $h \in H$ we have $\operatorname{card}(h(B) \triangle B)<\mathfrak{c}$.

3) For each Lebesgue measurable set $Z \subseteq \mathbb{R}^{2}$ with a strictly positive measure, the set $Z \cap B$ is of cardinality $\mathfrak{c}$.

Note that the construction of the set $B$ is analogous to the construction of the set $A$ mentioned above.

Now let $J$ be the family of all sets $Y$ such that there exists a covering of $Y$ by straight lines in $\mathbb{R}^{2}$ parallel to $\{0\} \times \mathbb{R}$, whose cardinality is strictly less than $\mathfrak{c}$.

Obviously, $J$ is an $H$-invariant $\sigma$-ideal of subsets of $\mathbb{R}^{2}$ such that $J+J=J$. Let $T$ be the $H$-invariant $\sigma$-algebra of subsets of $\mathbb{R}^{2}$ generated by the family $J \cup\{B\}$. Suppose that $\mathfrak{c}$ is a regular cardinal number. Then it is not difficult to check, starting with properties 1 ) and 2) of the set $B$, that for any subset $A$ of the line $\{0\} \times \mathbb{R}$ satisfying relations $(*)$ and $(* *)$, the vector sum $A+B$ does not belong to $T$. Hence, the relations $A \in J, B \in T$ and $A+B \notin T$ are fulfilled. It is also easy to see that the countable chain condition holds for the 
pair $(J, T)$ in a very strong form. Namely, if we take any three sets from $T \backslash J$, then at least two of these sets have a non-empty intersection.

Now consider $\mathbb{R}^{2}$ and $\mathbb{R}$ as vector spaces over the field $\mathbb{Q}$. Let $f: \mathbb{R}^{2} \rightarrow \mathbb{R}$ be an arbitrary isomorphism between these spaces. Let us put $I=f(J)$ and $S=f(T)$. Then $I$ is an invariant $\sigma$-ideal on the real line, $S$ is an invariant $\sigma$-algebra on the same line and the pair $(I, S)$ satisfies the countable chain condition in the strong form mentioned above. We also have $I+I=I$ and $I+S \neq S$. Thus, we conclude that the implication $S+S \neq S \Rightarrow I+I \neq I$ does not hold in some situations where the pair $(I, S)$ satisfies the countable chain condition. Moreover, in our case of $(I, S)$ we can define a complete probability measure $\mu$ on $S$ such that

(1) $\mu$ is invariant under the group of all isometric transformations of the real line;

(2) $\mu(f(B))=1 / 2$;

(3) $I$ coincides with the $\sigma$-ideal of all $\mu$-measure zero sets.

Remark 2. It is clear that all results considered in the paper remain true if we replace the real line $\mathbb{R}$ by an arbitrary uncountable vector space over the field $\mathbb{Q}$.

\section{References}

[1] J. C. Oxtoby, Measure and Category, Springer Verlag, Berlin, 1980.

[2] J. C. Morgan II, Point Set Theory, Marcel Dekker, Inc., New York and Basel, 1990.

[3] A. B. Kharazishvili, Invariant Extensions of the Lebesgue Measure, Izd. Tbil. Gos. Univ., Tbilisi, 1983, (in Russian).

[4] A. B. Kharazishvili, Some applications of Hamel bases, Bull. Acad. Sci. Georgian SSR, 85 no. 1 (1977), 17-20, (in Russian).

[5] W. Sierpinski, Sur la question de la mesurabilite de la base de M.Hamel, Fund. Math., 1 (1920), 105-111.

[6] S. Ruziewicz, Sur une propriete de la base hamelienne, Fund. Math., 26 (1936), 56-58.

[7] W. Sierpinski, Oeuvres Choisies, v. I - III, PWN, Warsaw, 1974-1976. 